\title{
3D Scene Reconstruction Using Multi-Sources Data
}

\author{
Mingder Yang ${ }^{1}$, Hungyu $\operatorname{Lin}^{1}$, Ilin Chan ${ }^{1}$, Huiping Tsai ${ }^{1, *}$ and Yiping Chen ${ }^{2}$ \\ ${ }^{1}$ Department of Civil Engineering, National Chung Hsing University, 145 Xingda Rd., Taichung 402, Taiwan \\ ${ }^{2}$ Department of Business Administration, Da-Yeh University, 168 University Rd., Dacun, Changhua, Taiwan \\ ${ }^{*}$ Corresponding author
}

\begin{abstract}
This paper focuses on 3D scene reconstruction by various simulation methods and multi-sources data. 3D scene reconstruction is a tool to be able to analyze an area by rendering it in 3D. This is a research piece that looks at how different methods of modeling have different functions and applications. The case study takes place in the Chung Hsing Lake at National Chung Hsing University (NCHU) campus. The findings of this research indicate the variety of techniques and methods used can reconstruct a 3D scene successfully. This research may help people in the future gain a basic understanding of the concepts of 3D scene reconstruction and establish $3 D$ scenes.
\end{abstract}

Keywords-3D scene reconstruction; image-based modeling (IBM); render; multi-sources

\section{INTRODUCTION}

3D Scene Reconstruction is the process of capturing the shape and appearance of real objects. The process of reconstructing a 3D scene requires many different techniques and much expertise to successfully bring all the methods together into a full scene reconstruction. 3D models, which can be constructed from remote sensors, have made huge advancements in recent years through both animated movies and games, as well as other industries where they have realized the huge potential advantages of 3D models for research and design [1].

Image-based modeling (IBM) uses many different viewpoints to reconstruct a real object on a computer. Models can be created using special image-taking devices that collect data about the location and orientation a photo is taken from to detect features. Alternatively, photos captured on an ordinary device (such as a smartphone) can be used in software to create a model. This second way of modeling requires photos to be taken at designed overlapping rate since the software has to detect the location and orientation the camera was in when the photos were taken using the information available from the image. David G Lowe (2004) presents a method that can be used to perform consistent feature detection between different views of an object or scene to extract coordinates from images [2]. This method allows for feature detection that is invariable to scale and rotation of the images, as well as still being able to detect features when there are different illumination and 3D rotation, across a large database of images taken from different locations. Lowe also describes more detail about feature match detection and the Hough Transform to dynamically identify objects using feature points found amid a cluttered background
[3]. In summary, the main steps for image-based modeling are as follows [4]:

- Image taking and motion tracking - images are taken of an object from multiple views whilst also tracking the distance and location of the camera relative to the object.

- Image processing, pattern recognition - import the images into computer software which uses the feature detection technique to find coordinates around the surface object. Tracking the distance and location of the camera relative to the object allows for more accurate results.

- Coordinate transformation and 3D reconstruction these coordinates can then be transformed using computer software to render a $3 \mathrm{D}$ replica for viewing.

Reconstructing a 3D scene provides a great way for people to view an area, location or object if they are unable to view it in person or if they need to view it from their location without traveling to the scene. Often 3D scene reconstruction will be able to present an area in a way that allows for better assessment than more traditional methods, such as technical drawings or $2 \mathrm{D}$ rendering. It is important to understand the process to be able to make the best use of $3 \mathrm{D}$ scene reconstructions.

The present study takes place in the Chung Hsing Lake at National Chung Hsing University (NCHU) in Taichung City, Taiwan. With approximately $7,000 \mathrm{~m}^{2}$ lake area, the Chung Hsing Lake has become an important recreation place for students and communities for more than 40 years.

\section{METHODOLOGY}

The process of $3 \mathrm{D}$ scene reconstruction is done using five computer programs including AutoCAD, SketchUp, PhotoScan, 3Ds Max, and Lumion (Figure I). To reconstruct the Chung Hsing Lake 3D scene model, three major modelings need to be done, which includes terrain modeling, geometric object modeling, and complex object modeling. Details of each modeling are described below. All the computer work was done on a computer running Microsoft Windows operation system with Intel ${ }^{\circledR}$ Xeon ${ }^{\circledR}$ CPU E3-1230 V2 @ 3.30GHz. For effective modeling, images were taken by a Canon PowerShot S110 digital high-resolution camera. 


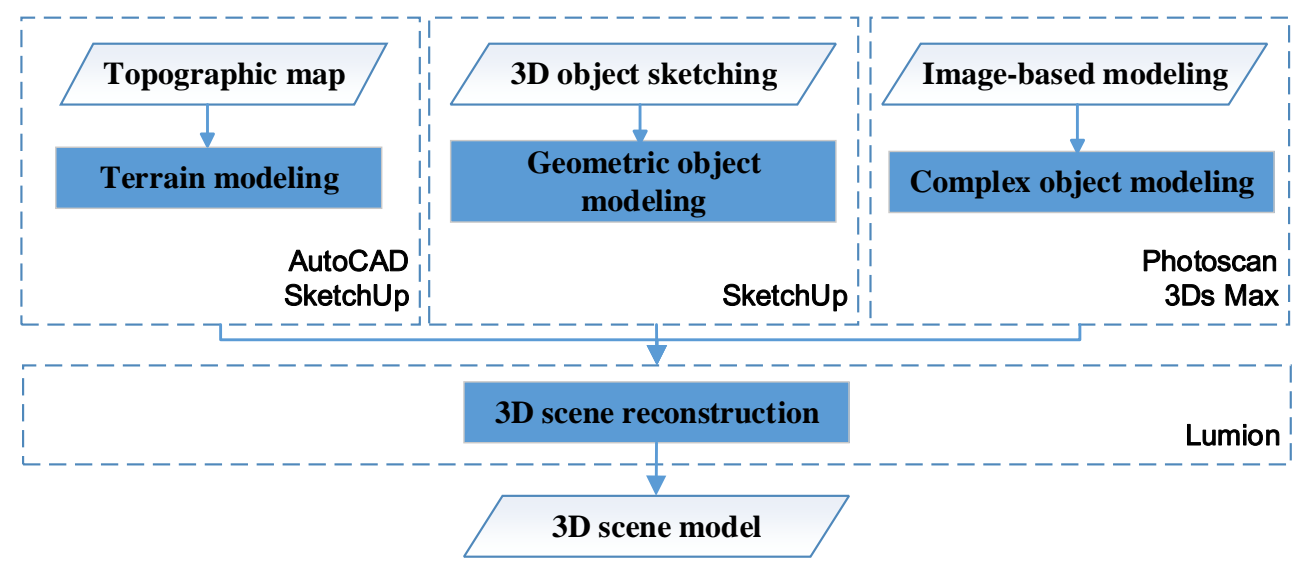

FIGURE I. FLOWCHART OF 3D SCENE RECONSTRUCTION PROCESS

The softwares used are listed below:

- AutoCAD [5] - is mostly used for terrain modeling.

- SketchUp [6] - creates simple models directly onto the computer.

- PhotoScan [7] - creates complex models through image-based modeling.

- $3 D s$ Max[8] - performs image inpainting process for 3D models.

- $\quad$ Lumion [9] - renders all imported models for the 3D scene reconstruction dislpay.

\section{A. Terrain Modeling}

For the 3D scene reconstruction, the first step involved is modeling the terrain, which is the dominant physical features of the land. This is done in three stages as shown in Figure II.

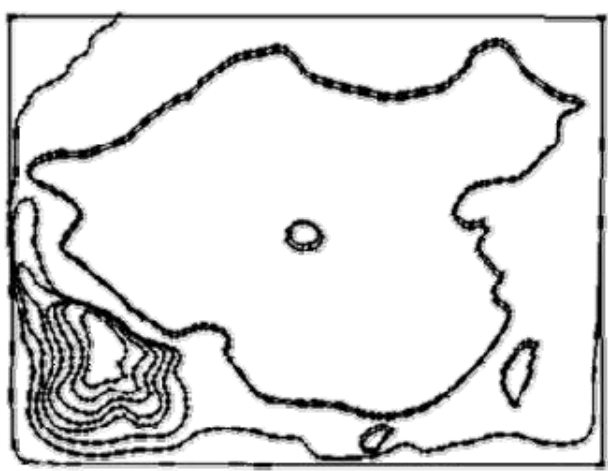

(A)

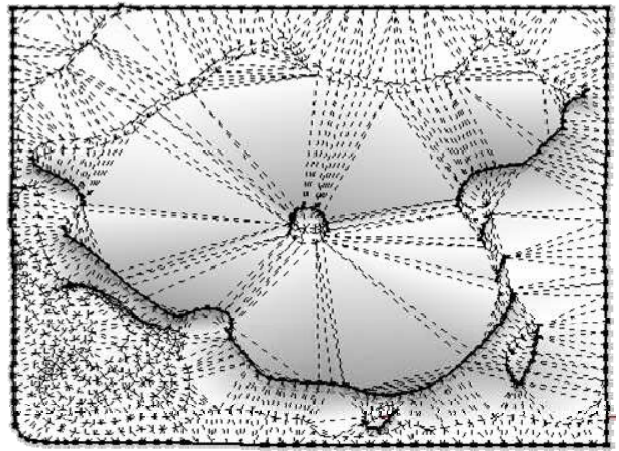

(B)

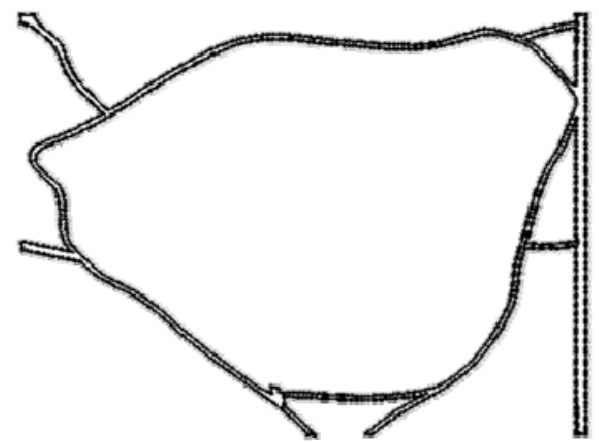

(C)

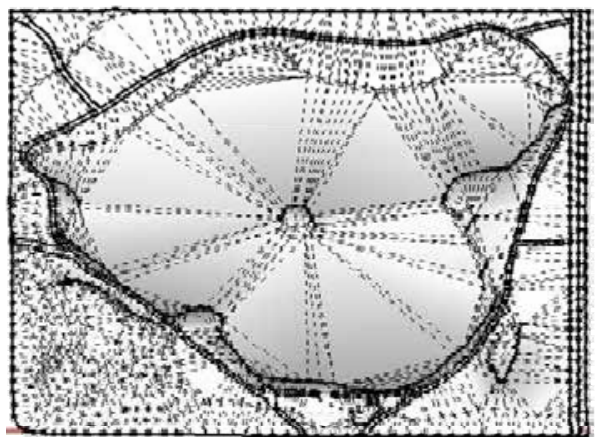

(D)

FIGURE II. TERRAIN MODELING STAGES: (A) CONTOUR LINES TAKEN FROM TOTAL STATION, (B)3D SURFACE FROM CONTOUR LINES, (C) LAYOUT OF ROAD FROM ABOVE, AND (D) FINAL TERRAIN MODEL WITH ROAD 
1) Digital elevation model: The digital elevation of the land is surveyed using a total station, which can detect angle, horizontal and vertical distance and coordinates along the terrain. This data can be stored internally and retrieved later or recorded directly onto an external data storage device. This data are then downloaded onto and imported into AutoCAD, which can plot the coordinates as contour lines along a plane.

2) 3D terrain modeling: This contour is then imported into SketchUp to create a 3D surface. SketchUp can automatically fill in this contour to be a 3D surface using the "Sandbox" function. AutoCAD is again used at this stage to create the layout of the surrounding road in a 2D format, using the bird's eye view of the location.

3) Terrain model: The final terrain modeling involves projecting the layout of the road onto the 3D surface created from the contours. Around Chung Hsing Lake, backgrounds and objects such as buildings were created later to give the scene of surrounding and context.

\section{B. Geometric Object Mmodeling}

The process of Geometric Object Modeling is all done directly into SketchUp. The process is briefly addressed as follows.

1) 3D object reconstruction: For $3 \mathrm{D}$ object reconstruction, a screenshot from Google Maps can be placed in SketchUp on the surface as a placeholder reference for objects, such as road divider and building position, that can serve as the found mental basis for further applications.

2) $3 D$ object sketch: Outlines are then sketched into SketchUp over the top of these positions. Google Maps can also be used to project the texture of buildings onto the surface. Small and simple objects can be done by hand drawing using estimations of position, and textures from SketchUp can be applied. These objects can be directly placed into the final model combined with other 3D background and objects.

\section{Complex Object Modeling}

Complex object modeling, including passive and active approaches, is used for objects which are too intricate to be modeled using geometric object modeling. In this case study, image-based modeling (IBM) was used to generate a statue of a scholar and a pavilion with appearance design in the shape of a mushroom. The IBM process was executed in software, PhotoScan. Photos must be taken of the model from many aspects to give multi-views and a best accuracy. 3D Max is used to perform image inpainting to repair broken parts of the 3D models [10]. For the scholar statue, 317 photos were taken as a large object with many fine details as Figure III, and the process is as follows:

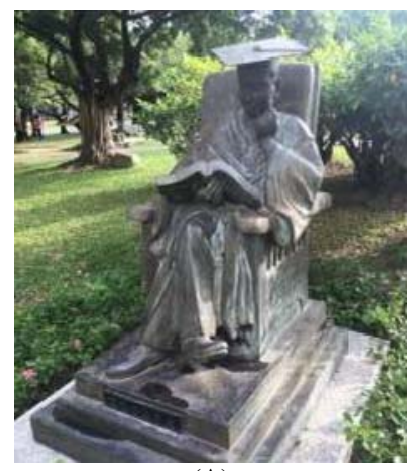

(A)

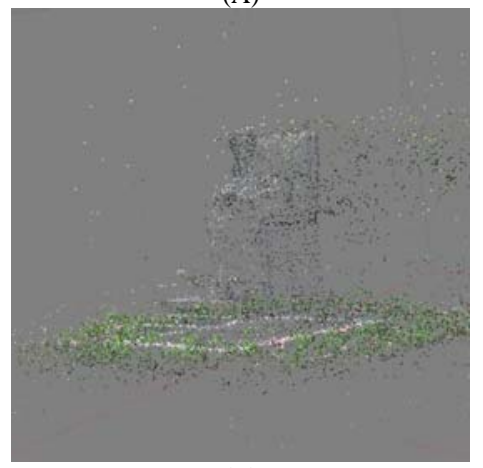

(B)

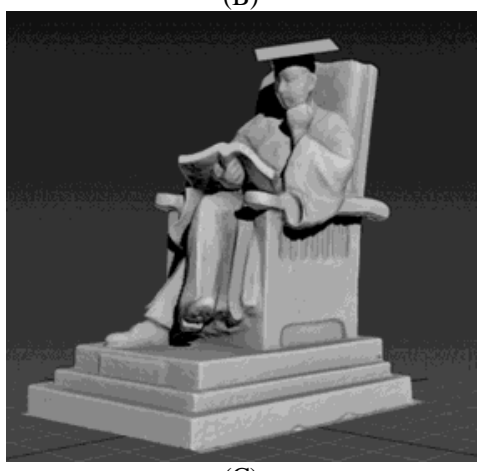

(C)

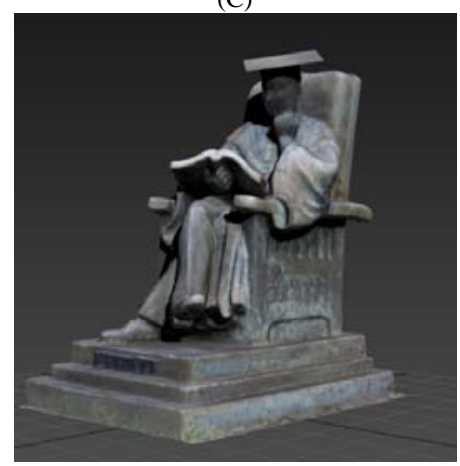

(D)

FIGURE III. COMPLEX OBJECT MODELING STEPS (SCHOLAR STATUE): (A) ORIGIN PHOTO, (B) POINT CLOUDS, (C) BUILDING MESH, AND (D) PROJECTING TEXTURE

- Photos were taken from various angles around the object.

- The photos are loaded onto PhotoScan, but do not yet have location or orientation information. 
- The photos are aligned to automatically estimate the location of where each photo was taken.

- A Dense Cloud is created which locates various points on the surface of the object.

- A “Mesh" links these various points to render a surface on the program.

- Once the surface is established, the texture can be projected on to the object.

\section{3D Scene Reconstruction}

When completing object modeling, the reconstructed 3D scene can be rendered in Lumion, which involves importing the various models from SketchUp and PhotoScan and correctly placing everything into the scene for the most accurate reconstruction. Once the objects are in place, more complex textures can be applied, as well as several other features seen in Lumion, such as a wind effect and the ability to view the scene in different illumination, for the effect of sunlight. Lumion can also be used to import common objects such as trees and rocks, and even people, into the 3D scene to interact with the actual environment and even imagination scenes.

\section{DISCUSSION AND CONCLUSION}

The results of 3D scene reconstruction of the Chung Hsing Lake are shown in Figure IV. At this stage, manual repair of the SketchUp terrain model is sometimes necessary to ensure significant errors being eliminated in the final 3D model. Of the three modeling types (terrain, geometric object and complex object), the complex object with high precision is the hardest and time-consuming method. The complex object method based on Image-Based Modeling requires many photos to be taken and its performance and precision highly depends on the imaging quality and environment. Additionally, the background, illumination, reference points, and image inpainting techniques need experience-based skill while reconstructing a 3D scene. Furthermore, the reconstructed 3D scene can be developed better and displayed in VR and AR environments.
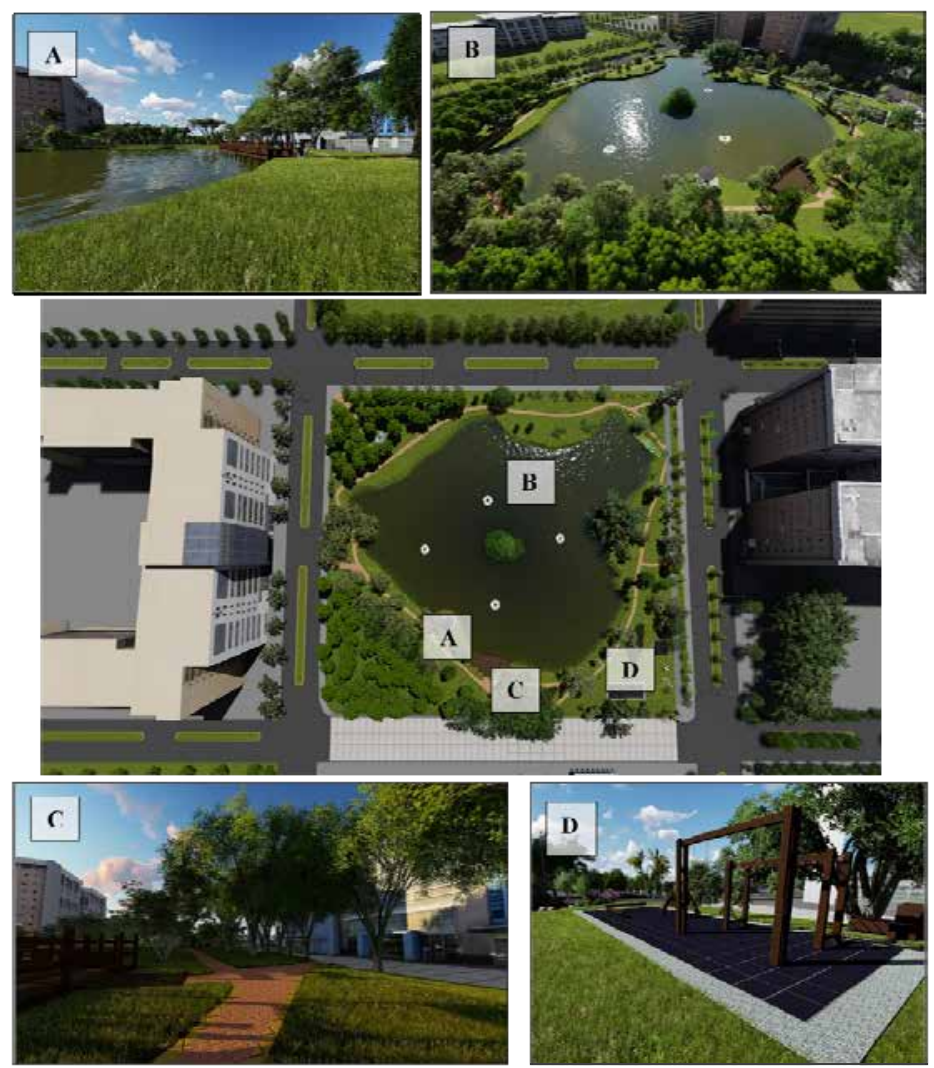

FIGURE IV. CHUNG HSING LAKE 3D SCENE MODEL

\section{REFERENCES}

[1] L. Karel, 2D and 3D Tracking and Modelling, ProQuest Dissertations Publishing, 2016.

[2] D. Lowe, "Distinctive image features from scale-invariant keypoints," International Journal of Computer Vision, vol.60(2), pp. 91-110, November 2004

[3] J. Illingworth, and J Kittler, "A survey of the Hough transform," Computer Vision, Graphics, and Image Processing, vol.43(1), pp. 87-116, October 1988.

[4] M.D. Yang, C.F. Chao, L.Y. Lu, K.S. Huang, and Y.P. Chen, "Imagebased 3D scene reconstruction and exploration in augmented reality," Automation in Construction, vol.33, pp. 48-60, August 2013.

[5] AutoCAD, AutoDesk, Information https://www.autodesk.com/education/free-software/autocad

[6] Sketchup, Trimble, Information on https://www.sketchup.com/

[7] Photoscan, Agisoft, Information http://www.agisoft.com/downloads/installer/

[8] 3Ds Max, AutoDesk, Information https://www.autodesk.com/products/3ds-max/overview

[9] Act-3D, Lumion, Information on https://lumion3d.com/

[10] K.R. Patel, L. Jain, and A.G. Patel, "Image Inpainting - A review of the underlying different algorithms and comparative study of the inpainting techniques”. International Journal of Computer Applications vol.118, pp. 32-38, May 2015. 